East African Medical Journal Vol. 85 No. 9 September 2008

DELIVERING NEW MALARIA DRUGS THROUGH GRASSROOTS PRIVATE SECTOR

A. N.Chiguzo, Dip, MSc, Malaria Project Coordinator, Exxon Mobil Project. Sustainable Healthcare Foundation, R. W. Mugo, MBChB, MPH, Health Services Manager, Sustainable Healthcare Foundation, $1^{\text {st }}$ Ngong Avenue, ACK Garden House, Wing A, First Floor, P. O Box 1630, 00606 Nairobi, Kenya, D. G. Wacira, MPH, PhD, Malaria Programme Manager, African Medical and Research Foundation, P. O. Box 30125, Nairobi, Kenya, E. W. Njuguna, BSc, MBA, SHF's Executive Director, J. M. Mwenda, Dip. in Pharmacy, SHF's Pharmaceutical Technologists of the Exxon Mobil Project, Sustainable Healthcare Foundation, $1^{\text {st }}$ Ngong Avenue, ACK Garden House, Wing A, First Floor, P.O Box 1630, 00606 Nairobi, Kenya

Request for reprints to: Mr. A. N. Chiguzo, Sustainable Healthcare Foundation, $1^{\text {st }}$ Ngong Avenue, ACK Garden House, Wing A, First Floor, P.O. Box 1630, 00606 Nairobi, Kenya

\title{
DELIVERING NEW MALARIA DRUGS THROUGH GRASSROOTS PRIVATE SECTOR
}

\author{
A.N. CHIGUZO, R. W. MUGO, D. G. WACIRA, J.M. MWENDA \\ and E.W. NJUGUNA
}

\begin{abstract}
Objective: To demonstrate that micro-franchising system is an effective way of improving access to effective health care such as the introduction of first line antimalarias in populations living in underserved rural areas in Kenya.

Design: A descriptive study.

Setting: Child and family wellness (CFW) micro-franchised nurse run clinics in Kenya.

Results: In 2007, 39.3\% of RDTs carried out were positive for malaria. All malaria positive (RDTs and microscopy) patients received artemether lumefantrine (AL) according to their weight in accordance with the Government approved treatment guidelines. During the same period a total of 3,248 community members were reached with malaria information, however, community expectations took longer to change as patients demanded AL even when the malaria diagnosis was negative. Initially, this led to the dispensing of other antimalarials to patients with malaria like symptoms even with a negative test. This demand decreased with more community education on the importance of the tests. Engaging the private sector though with challenges proved feasible and appropriate in accessing malaria treatment based on clinical diagnosis supported by RDTs to confirm the diagnosis instead of presumptive treatment based on fever. This led to a reduction of antimalarial prescriptions by more than $50 \%$, implying better patient care, rational drug use as well as cost savings on malaria treatment. Conclusion: A micro-franchising system is an effective and sustainable way of improving access to effective health care by populations living in underserved rural areas of Africa. With appropriate supportive training and supervision, the system can adapt to changes in treatment guidelines and to new regimens.
\end{abstract}

\section{INTRODUCTION}

Sustainable Healthcare Foundation (SHF) is a notfor-profit organisation whose mission is to prevent needless death and illnesses through sustainable methods of improving access to essential drugs and basic health services for children and families in the resource scarce communities. SHF applies a solid business solution (franchising) to a vast public sector problem; it has adapted and refined the franchising model to deliver critical healthcare services to underserved populations. SHF recruits franchisees with previous health experience and takes them through standardised rigorous training, and applies strict quality control systems for consistency across the outlets. Those outlets that consistently under perform are revoked and replaced with new outlets (1). The programme started in 1999 with 
11 drug shops and is currently running 65 outlets in ten districts of Kenya. The programme is being expanded to reach 225 outlets by 2012 . The expanded programme will adopt new and more effective high quality interventions such as, access to basic health care, access to essential medicines, school health promotion safe water promotion, growth monitoring, and community education.

In the distribution of AL through the child and family wellness (CFW) health providers, SHF adheres to the stipulated government recommendations (Figure 1).
Currently our reach is 65 outlets and we hope to reach 225 outlets in the next five years. The nurse-run clinics provide essential medicines and basic health care to approximately 400,000 people annually (3). Malaria is the commonest cause of illness, followed by upper respiratory tract infections (URTI), diarrhoea, intestinal worms and skin conditions. Malaria prevalence among patients who seek treatment at the clinics is estimated at $40 \%(1,3)$.

In the past twelve months SHF has been piloting a project to provide access to first line antimalarial medicines using ACTs in nine nurse-run CFW clinics

Figure 1

Proposed AL use strategy

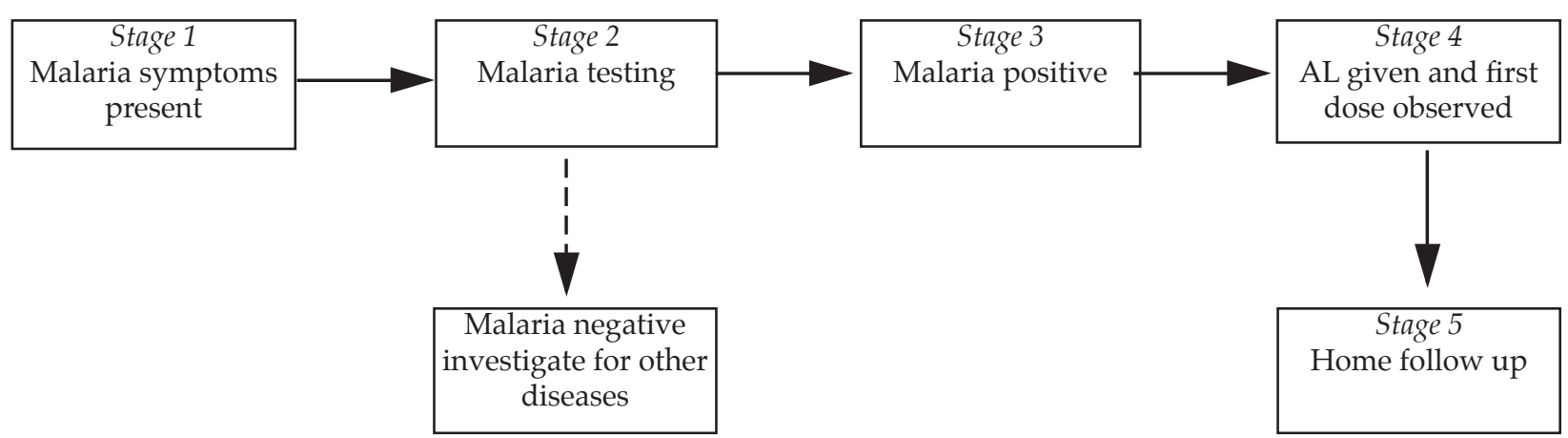

NOTE: The testing is mostly recommended for children above five years and adults, for children under five years, testing is not a prerequisite for treatment but desirable where RDTs are available especially in low malaria endemic areas (2).

This recommendation recognises the need to prevent or delay potential drug resistance that may arise, prevent drug abuse or misuse and also ensure adherence to the prescriptions. In line with the $\mathrm{MOH}$ requirements, a monitoring and evaluation system is in place to ensure that the guidelines are adhered to (2).

AL is administered as a six dose regimen. The health provider explains the dosing schedule, and ensures the patient takes the first treatment dose at the clinic. The patients are counselled on malaria prevention, the need to return for review and to report any adverse reactions.

The grassroots private sector has a key role in the delivery of lifesaving medicines and other interventions, but engaging them requires innovative approaches and careful assessment.

Sustainable Healthcare Foundation (SHF) has for the past seven years pioneered the application of an effective business format franchise model to distribute essential drugs and basic healthcare in rural Kenya. with support from Exxon Mobil Foundation. This pilot project operates using four defined protocols namely, patient service, data collection, training and community education.

The role of medicine retailer programmes in the Kenyan National Malaria Strategy: In recent years, Kenya has experienced a number of initiatives aimed at improving service delivery of anti-malarial medicines using different approaches $(4,5)$. The rationale for these initiatives is based on widespread recognition of the important role that private medicine retailers play in providing first line treatment for a variety of medical conditions in Kenya, including childhood fevers $(6,7)$. The 2001 Kenya National Malaria Strategy recommends such programmes as a means of improving early access to effective antimalarial treatment for children with fever (8).

Between 2002 and 2005, the Division of Malaria Control (DoMC) in the Ministry of Health (MOH) supported 30 districts to obtain funding through the Global Fund for AIDS, TB and malaria (GFATM) to implement drug retailer programmes. A temporary working group in the DoMC developed guidelines for the implementation of medicine retailer programmes at district level in 2002 (9). On the basis of these guidelines, a research team from the Kenya 
Medical Research Institute (KEMRI)-Wellcome Trust Programme has been working in collaboration with the DoMC and District Health Management Teams (DHMT) in three districts (Busia, Kwale and Makueni) to provide initial support for implementation and to evaluate medicine retailer programmes implemented between 2002 and 2005 (10).

In 2004, the DoMC recommended a new antimalarial drug policy to the Government of Kenya. The change concerned first line antimalarials (AM) for non-severe malaria, comprising a shift from the failing sulphadoxine pyrimethamine (SP) antimalarials to artemesinin based combination therapy (ACT). Initially this was to be implemented in the formal sector (government clinics and hospitals), followed by the formal private sector (private clinics and hospitals) and lastly in the informal retail sector (general sales) if experience and research supported this last phase and indicated appropriate mechanisms. In view of the failure rates seen for SP drugs in many parts of Kenya, the DoMC identified amodiaquine (AQ) antimalarials as the most appropriate over-thecounter (OTC) preparations for first line treatment of malaria in the interim period. The retailer programme evaluations were therefore based on indicators related to AQ sales and use (10).

\section{MATERIALS AND METHODS}

The pilot project has four main components. (i) Training of nurses running the rural clinics on drug dispensing according to the new malaria treatment guidelines, malaria diagnosis using rapid diagnostic test and record keeping. (ii) Community education on care seeking for malaria, compliance to treatment and malaria prevention. (iii) A patient service component which involves history taking, basic physical examination, performing malaria diagnosis, dispensing of the new antimalarial artemether lumefantrine (AL) to malaria positive patients.

Patients who do not have malaria are examined to establish other causes of fever and are either treated by the nurses or referred to nearby health facilities with more qualified personnel as well as laboratory facilities. Patients are followed up to monitor recovery and for adverse drug reactions. (iv) Monitoring through routine record keeping and community surveys.

\section{Patient protocols}

(i) The patient is greeted, welcomed and led to the consultation room.

(ii) Personal details are taken.

(iii) Vital observations are taken: blood pressure, pulse, temperature, respiratory rate and weight

(iv) Weightisimportant asitgives guidanceondrug dosing. In the case of malaria, it will determine the dose category of AL to be used (doses are grouped into weight bands of $5-14 \mathrm{~kg}, 15-24$ $\mathrm{kg}$, 25-34 $\mathrm{kg}$ and $35+\mathrm{kg}$ ).

(v) Medical history is taken, noting the presenting complaint, history of the present complaint, past medical history, family and social history.

(vi) A physical examination is carried out with the patient on the couch.

(vii) The clinician then makes diagnosis or tentative diagnosis using the history and physical examination findings and informs the patient of the same and the need for confirmatory tests.

(viii) In case of a tentative diagnosis of malaria, a rapid diagnostic test (RDT) is carried out and the result read after 15 minutes. If the test indicates one has malaria, AL is issued and patient advised on the dose, duration and frequency of taking the drug. The first dose is observed at the clinic, with demonstrations for the patient on how to take the drug and in the case of a child, how to crush and mix with clean water. Paracetamol for pain and fever is also issued.

(ix) If a patient is too sick to be handled in the outlet, basic history and observations are taken and some first aid offered. A referral form is then filled and relatives directed where to take the patient.

(x) All patients are advised on a review date which is indicated on a TCA (To Come Again) card as well as report on any adverse drug reactions (ADR) (Pharmacovigilance).

(xi) Patients with ADR associated with AL are managed as per the manufacturer's recommendations.

(xii) Patients with severe malaria are managed as per the recommendations in the malaria treatment guidelines.

Data collection protocols: Figure 2 outlines the tools developed to collect data for monitoring project activities which are reported monthly from each outlet:

Figure 2

Tools developed to collect data for monitoring project activities

- Daily patient register (DPR).

- AL requisition form as per weight categories.

- AL stock card as per weight categories.

- AL consumption form as per weight categories.

- RDT stock cards.

- RDT requisition forms.

- RDT consumption forms.

- Malaria specific morbidity form separated to $<5$ and $>5$ age categories.

- Pharmacovigilance on adverse drug reactions (ADRs) form. 
Training protocols: A team consisting of SHF's malaria project coordinator, health services manager, training and communication manager and health services officers have been trained as ToTs by Ministry of Health on the new $\mathrm{MOH}$ training guidelines for diagnosis and treatment of malaria. This team conducts a three day residential training for nurses from the outlets. The training covers the following topics:

(i) New treatment guidelines.

(ii) Malaria diagnosis.

(iii) Theory and practicals on carrying out the rapid diagnostic tests.

(iv) Theory on malaria microscopy.

(v) Dispensing of AL/Pharmacovigilance.

(vi) Health education.

(vii) Record keeping.

Then, a one-day residential continuing education session is conducted after three months of implementation. The session includes, but is not limited to, the following topics:

(i) Project progress update.

(ii) Experience sharing.

(iii) Areas of the project that need emphasis.

(iv) Challenges and how to overcome them.

Community education protocols: In rolling out community education, SHF has developed scripts and information, education and communication (IEC) materials with messages and information on malaria treatment, prevention and control. SHF identifies comedian/theatre groups within the outlets that have experience in developing malaria messages into songs, drama and poems. These groups are used to perform once every quarter at public meetings (Chief's barazas) to educate the community in their vernacular about malaria. The theme of the community education is "Stop malaria...Save life... use AL. Follow dosage regimen of AL to completion for effective cure of malaria."

During these public meetings or Chief's Barazas the following is carried out:

(i) Talks on malaria/AL.

(ii) Skits on malaria/Al.

(iii) Songs on malaria/Al.

(iv) Poems on malaria/AL.

(v) Posters, leaflets and fliers on AL are distributed.

(vi) Caps and t-shirts with AL messages are distributed.

(vii) Messages--"Komesha Malaria... Okoa Maisha, Tumia AL" (Stop malaria... Save Life... Use AL).

(viii) Distribution of IEC materials in the form of calendars, fliers, brochures, leaflets and posters, t-shirts and caps.
Other channels for community education that SHF requires every franchisee to use include, but are not limited to, the following:

(i) Outreaches/home visits.

(ii) One on one.

(iii) Schools.

(iv) Mosques/Churches.

(v) Organised community group meetings.

\section{RESULTS}

Parasitological baseline survey: In order to determine the malaria prevalence at the project sites, a parasitological survey was carried out at 17 different CFW clinics in 17 market centres in three districts of SHF's central region. The central region serves Kirinyaga, Embu and Mbeere districts of Kenya. A community mobilisation exercise for children under five was conducted with assistance from $\mathrm{MoH}$ staff and the Provincial administration.

Blood samples were then taken from 2744 children aged 6-59 months and examined for malaria by experts from the Division of Vector Borne Diseases (DVBD) in Mwea. As an incentive, every child got a free ITN, while those who were sick got free treatment.

The survey was carried out in the months of October and November 2006, which represents a low malaria transmission season. Project sites that had at least one positive blood slide for malaria parasites were selected. A positive malaria slide detected in a site was indicative of malaria presence as well as active malaria transmission.

Findings of the parasitological survey: The survey confirmed active malaria transmission in, Kevote, Ugweri, Gatunduri, Nthagaiya and Ena markets in Embu district, of which all were selected as pilot sites. Ugweri market had the highest blood positivity rate among children aged 6-59 months.

Selection of project sites: Project sites were selected based on: past malaria morbidity, malaria disease burden (after a parasitological survey to determine malaria prevalence), capacity of the franchisee to assimilate new products, and current business performance of the clinics. Ten outlets were selected which include five from Embu, three from Kirinyaga and two from Mbeere districts. AL was obtained from Kenya Medical Supplies Agency (KEMSA) through Division of Malaria Control (DoMC) on a quarterly basis and availed to the user free of cost while Rapid Diagnostic Tests (RDTs) were purchased through SHF procurement systems. RDTs were charged to the clients at a subsidised fee of Ksh 20.

In order to improve the tracking system, and as required by $\mathrm{MoH}$ because AL is a prescriptiononly drug, the project hired two pharmaceutical 
technologists to strengthen capacity of dispensing AL, monitor and manage ALas well as pharmacovigillance for adverse drug reaction (ADR).

Community education: In rolling out community education, several channels were used including public meetings (Chief's barazas), outreach/home visits, cultural theatre groups, schools and mosques / churches. The theme of the community education is "Stop Malaria...Save Life. Follow dosage regimen of AL to completion for effective cure of malaria".
Tostrengthencommunity educationandoutreach activities, two nurses were hired. Community education was carried out at several public meetings (Chiefs' barazas) within the project site.

A total of 3,248 people were reached with education at barazas through the nine outlets. On average, at least two community meetings were held at each of the nine outlets.

Table 1

Selected project sites

\begin{tabular}{llll}
\hline Name of CFW Clinic & Locality/market & District & Franchisee \\
\hline Tumaini Clinic* & Ugweri & Embu & Mary Njue \\
By Grace Clinic* & Gatunduri & Embu & Mary Njeru \\
Tender loving Care* & Kevote & Embu & Tasiana Mateo \\
Msamaria Mwema* & Nthagaiya & Embu & Reuben (Mercy) \\
Njiwa Clinic* & Ena & Embu & Jeane Kathangai \\
Njuka Clinic* & Kiorugari & Kirinyaga & Daniel Kamanga \\
Huruma Clinic & Kiritiri & Mbeere & Rosemary M. Njagi \\
Arahuka Shop & Kiandegwa & Kirinyaga & Isabella Maina \\
St. Judy Shop & Gatwiri & Kirinyaga & Catherine Mutava \\
Karaba Shop & Karaba & Mbeere & Jane Kiswili \\
\hline
\end{tabular}

* CFW clinics in the first phase of the study

Table 2

Distribution and supply of AL and RDTs to project sites, December 2006-November 2007 CFW clinics in the first phase of the study

\begin{tabular}{llrrrrrrr}
\hline \multirow{2}{*}{$\begin{array}{l}\text { Name of } \\
\text { CFW Clinic }\end{array}$} & Locality/ & \multirow{2}{*}{ RDT } & \multicolumn{3}{c}{ Quantities of AL issued } & \multicolumn{2}{c}{ Total } \\
& market & issued & $5-14$ & $15-24$ & $25-34$ & $35+$ & AL & $(\%)$ \\
\hline Tumaini & Ugweri & 1900 & 300 & 120 & 120 & 900 & 1440 & 31.05 \\
By Grace & Gatunduri & 150 & 60 & 60 & 30 & 60 & 210 & 4.53 \\
T L C & Kevote & 600 & 180 & 120 & 30 & 150 & 480 & 10.35 \\
Msamaria & Nthagaiya & 350 & 60 & 30 & 60 & 180 & 330 & 7.12 \\
Njiwa & Ena & 700 & 90 & 60 & 30 & 150 & 330 & 7.12 \\
Njuka & Kiorugari & 1050 & 180 & 120 & 86 & 450 & 836 & 18.03 \\
Huruma & Kiritiri & 475 & 90 & 60 & 60 & 60 & 270 & 5.82 \\
Arahuka & Kiandegwa & 250 & 90 & 60 & 30 & 141 & 321 & 6.92 \\
St. Judy & Gatwiri & 325 & 120 & 60 & 30 & 210 & 420 & 9.06 \\
\hline & Total & 5825 & 1170 & 690 & 476 & 2301 & 4637 & 100 \\
\hline
\end{tabular}


Tumaini and Njuka clinics demanded more RDTs and AL because they had more clients and were located in high malaria endemic areas. This shows the importance of having update attendance record to determine the supplies for a push system.

Initial supplies were based on a push system to the project sites but subsequent supplies are being replenished based on a pull system (demand from the project sites).

Preliminary findings from this project show that a total 5,584 suspected malaria cases were tested at the project sites between December 2006 and November 2007. Out of these, 2,195 (39.3\%) were RDT positive while 412 were blood slide (BS) referral positive and all were treated for malaria with AL. By introducing definitive diagnosis for malaria (using RDTs) in our setting, we estimate $53.4 \%$ cost savings on AL compared to presumptive diagnosis (clinical).
Figure 3 shows progressively higher percentages of positive malaria cases. This could either be due to increase in cases or due to improved diagnosis following community outreach.

Figure 4 shows the AL consumption trends based on the various patient weight ( $\mathrm{kgs}$ ) categories. The highest consumption was by those weighing $35 \mathrm{kgs}$ and above followed by the $5-14 \mathrm{kgs}$ category. The lowest was the $25-34 \mathrm{~kg}$ category. Arahuka and St. Judy clinics started their activities in March 2007 as they were in the second phase

\section{DISCUSSION}

Malaria prevalence among patients (both the $<5^{\prime}$ s and $>5$ 's) who had fever or were suspected to have malaria was about $40 \%$ (2195 out of 5584). These are the patients who tested positive for malaria. All

Figure 3

Malaria RDT positivity rate December 2006 to November 2007

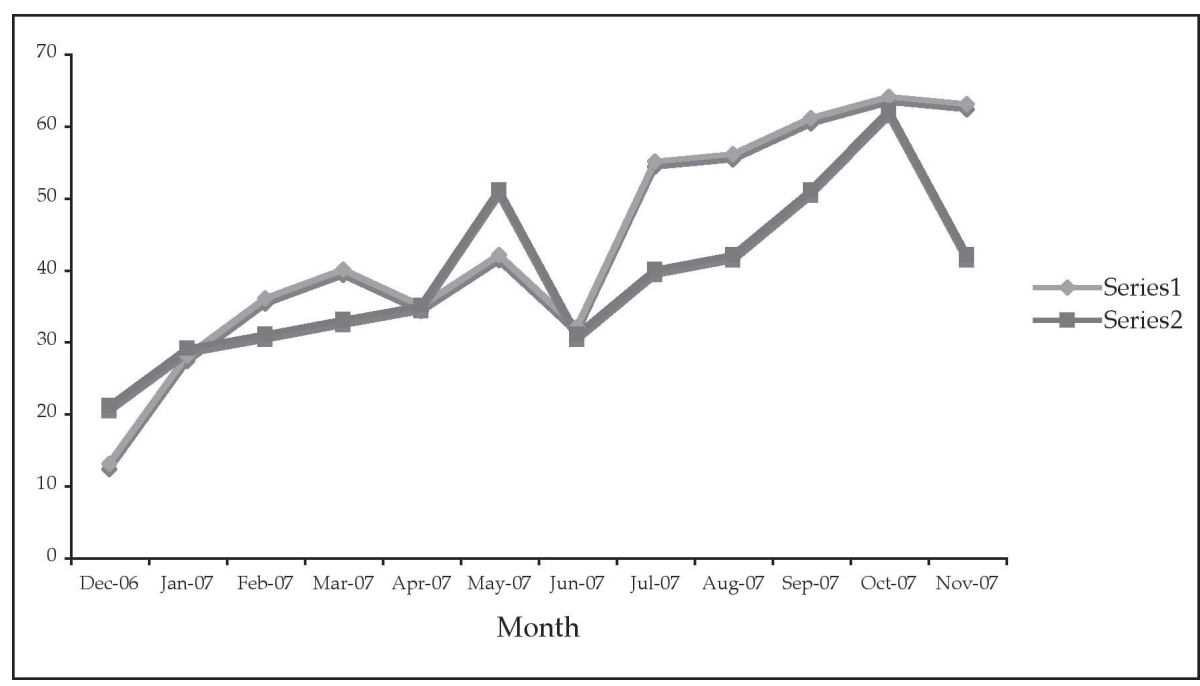

Figure 4

AL consumption among positive malaria cases by category

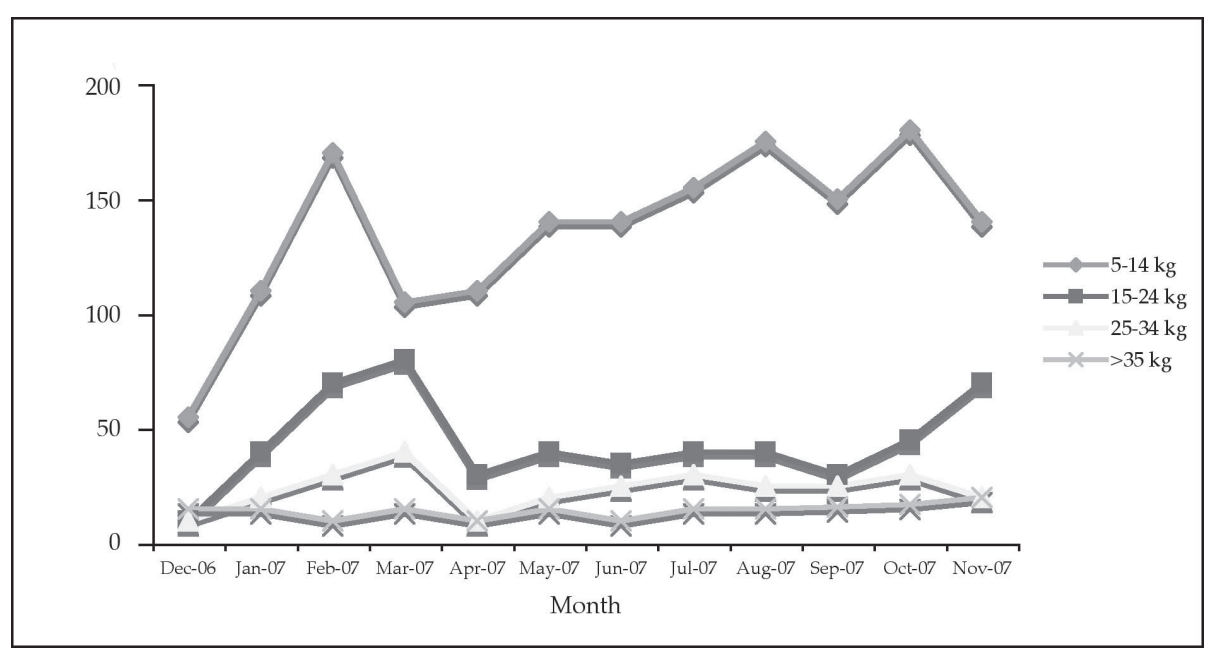


malaria positive patients received the right dose of $\mathrm{AL}$ according to their body weight. The most commonly used AL category was that of children and adults $>35$ kilograms. This was followed by the $5-14 \mathrm{~kg}$ category comprising mainly of the under fives an age group that is highly susceptible to malaria. The lowest was the $25-34 \mathrm{~kg}$ category made up of adolescents. This implies that when ordering for $\mathrm{AL}$, more quantities of the category of $35+\mathrm{kg}$ were required while the category of 25-34 kg required fewer quantities. In view of the above and formerly as shown in Table 2 , it is important to have accurate data to enable the project to place orders for the requisition and distribution of AL and RDTs to the clinics. Three thousand two hundred and fourty eight community members were reached with malaria information; however, community expectations took longer to change as patients demanded AL even when the malaria diagnosis was negative. Initially, this led to the dispensing of other antimalarials to patients with malaria like symptoms even with a negative test. This demand decreased with more community education on the importance of the tests.

Engaging the private sector proved to be feasible and appropriate in malaria treatment based on diagnosis instead of presumptive treatment based on fever, reducing the use of expensive antimalarials by more than $50 \%$, implying better patient care and cost savings on malaria treatment.

Over the twelve months, only two cases of adverse drug reactions (ADR) were reported in Njuka and Tumaini clinics. In the former a child experienced oral thrush after three days of taking AL. This could not directly be related to $\mathrm{AL}$, other concomitant infections or drugs might have been responsible. The patient in Tumaini developed pruritus which is one of the side effects documented by the drug manufacturer. The patient who developed pruritus discontinued AL treatment on day two and was given amodiaquine for three days while the one who developed oral thrush was put on clotrimazole oral paint for five days and both responded well.

The current project is scheduled to expand to all the CFW clinics starting from mid 2008-2010 and serving hundreds of thousands of malaria victims every year. We believe that full deployment of this programme throughout an expanded network of clinics will significantly magnify its impact and that it could become the most effective malaria project of its kind in Africa.

Thenurse-runclinics provideessential medicines and basichealth care to approximately 400,000 people annually. Malaria is the commonest cause of illness. Exxon Mobil has been a valued partner in these first steps toward a better model for the sustainable provision healthcare in the developing world. We look forward to a continuing partnership with Exxon Mobil for a long time to come.
In conclusion, a micro-franchising system is an effective and sustainable way of improving access to effective health care by populations living in underserved rural areas of Africa. With appropriate supportive training and supervision, the system can adapt to changes in treatment guidelines and to new regimens.

\section{ACKNOWLEDGEMENTS}

To Exxon Mobil Foundation for the financial support of this study. We would wish to thank the various Franchisees involved in the project for their support in data collection. We are grateful to Sustainable Healthcare Foundation for allowing use of their vehicle in collection of part of the data on which this analysis was based. Special thanks to Njoroge Muigai and Muthee Mwangi of the Division of Vector Borne Diseases (DVBD) Mwea for their support in parasitological baseline survey.

\section{REFERENCES}

1. Steadman Group. Evaluating the introduction of Artemether Lumefantrine in selected clinic operating under CFW Franchising System in Kirinyaga, Embu and Mbeere districts. WHO Baseline Report. 2007.

2. Ministry of Health. National guidelines for diagnosis, treatment and prevention of malaria for health workers in Kenya. Division of Malaria Control, Ministry of Health. 2006.

3. Sustainable Healthcare Foundation. Using microfranchising to improve access to life-saving medicines. A proposal to expand access to effective antimalaria medicines by introducing coartem into selected CFW clinics in Kirinyaga, Embu and Mbeere districts, 2005.

4. Tavrov, P. Shabang, J. and Makama, S. Vendor to vendor education to improve malaria treatment by private drug outlets in Bungoma district, Kenya. Malar. J. 2003; 2:10.

5. Marsh, V., Mutemi, W., Willtes, A., et al. Improving malaria home treatment by training drug retailers in rural Kenya. Trop. Med. Int. Health. 2004; 9: 451-460.

6. Snow, R. W., Peshu, N., et al. The role of shops in the treatment and prevention of childhood malaria on the coast of Kenya. Trans. R. Soc. Trop. Med. Hyg. 1992; 86: 237-239.

7. Amin, A. A., Marsh,V., et al. The use of formal and informal curative services in the management of paediatric fevers in four districts in Kenya. Trop. Med. Int. Health. 2003; 8: 1143-1152.

8. Ministry of Health. National Malaria Strategy 20012010. 2001.

9. Ministry of Health Report on the retail sector and malaria control working group, 2002.

10. Abuya, T., Rowa, Y., Kombe, F. et al. Final report on District-led medicine retailer training programmes in Busia, Kwale and Makueni districts. 2006. 